\title{
APPROXIMATION THEOREMS ON DIFFERENTIABLE SUBMANIFOLDS OF A COMPLEX MANIFOLD
}

\author{
BY \\ RICARDO NIRENBERG AND R. O. WELLS, JR.(1)
}

1. Introduction. Let $X$ be a complex manifold, $\mathcal{O}$ be the sheaf of germs of holomorphic functions on $X$, and $\mathcal{O}(S)(=\Gamma(S, \mathcal{O}))$ be the sections of $\mathcal{O}$ over $S \subset X$ (the holomorphic functions on $S$ ). If $K$ is compact in $X$, let $C(K)$ denote the Banach algebra of continuous complex-valued functions on $K$ with the maximum norm. There is a natural restriction map $r: \mathcal{O}(K) \rightarrow C(K)$, and an old problem in complex analysis has been to determine the closure of the image of $\mathcal{O}(K)$ in $C(K)$, under various conditions on $X$ and $K$.

Classically $X$ was the complex plane, and a well-known theorem of Mergelyan asserts that: if $K$ has no interior and the complement of $K$ has only a finite number of components, then $\mathcal{O}(K)$ is dense in $C(K)$ (in fact more is true, see the survey paper by Wermer [19]). Similarly, if $K$ is the closure of a nice domain $D \subset C$, then it follows from Mergelyan's results that the closure of $O(K)$ in $C(K)$ consists of all functions holomorphic in $D$ and continuous on $\bar{D}$.

In $C^{n}$ and on complex manifolds in general things are not quite so simple, and here the geometry of the compact set $K$ plays a crucial role. For instance, if $K$ is a compact arc or a closed curve (differentiable and otherwise) in $\boldsymbol{C}^{n}$, extensive investigations concerning the approximation of continuous functions on $K$ by polynomials or rational functions have been made, using techniques which stem from function theory in one variable and function algebras on the unit disc (see [19], [13]).

In this paper we use techniques of partial differential equations, and, in particular, the solution to the $\bar{\partial}$-Neumann problem, to derive some general approximation theorems on $C^{\infty}$ submanifolds of a complex manifold. Our principal result (Theorem 6.1) is that any compact $C^{\infty}$ submanifold $M$ of a complex manifold $X$ with the property that $M$ is totally real (that is, $M$ has no holomorphic tangent vectors, see $\$ 2$ for a precise definition) has an open Stein neighborhood $U$ in $X$ such that the natural map $O(U) \rightarrow C^{\infty}(M)$ is dense in the $C^{\infty}$ topology on $M$. In particular any curve $\left(C^{\infty} 1\right.$-manifold) automatically has this property and other examples are given in $\S 2$. This result implies trivially that $\mathcal{O}(U)$ and $\mathcal{O}(M)$ are dense in $C(M)$, and this gives a generalization of a weak form of Mergelyan's theorem to higher dimensions, where the totally real condition is the natural generalization (in the smooth manifold case) of the "no interior" condition in the plane. There are simple examples to show that the totally real condition is necessary in general.

Received by the editors February 27, 1968.

(1) Research supported by Army DA-ARO-D-31-124-G866 and NSF GP-5951. 
Using the same techniques we are able to prove various related results. We show that for a suitable type of pseudoconvex domain $D$ in $C^{n}$ with a smooth boundary, the mapping $\mathcal{O}(\bar{D}) \rightarrow \mathcal{O}(D) \cap C^{\infty}(\bar{D})$ is dense $\left({ }^{2}\right)$, which does not imply that the map $\mathcal{O}(\bar{D}) \rightarrow \mathcal{O}(D) \cap C(\bar{D})$ is dense, this being still an open problem for domains $D$ with a smooth boundary. Our result thus gives a partial answer to this old problem.

If we remove the totally real hypothesis from a submanifold $M$, then one can ask which functions on $M$ can be uniformly approximated by holomorphic functions defined near $M$. In this direction we show that if $M$ is a $C^{\infty}$ fibred family of complex submanifolds of $C^{n}$ (the Levi form on $M$ is $\equiv 0$ ), and $f$ is a $C^{\infty}$ function on $M$, holomorphic on the fibres, then $f$ can be locally (uniformly) approximated by holomorphic functions in $C^{n}$ (Theorem 6.6). A somewhat harder result which we can prove only for hypersurfaces is that any $C^{\infty}$ function $f$ on a real hypersurface in $C^{n}$ and satisfying the induced Cauchy-Riemann equations can be locally approximated by holomorphic functions (Theorem 6.5). We have been unable to prove a similar global result for hypersurfaces or for submanifolds of lower dimension.

In $\S 7$ we apply the theorems of $\S 6$ to prove some global approximation theorems, generalizing results of Wermer ([19], [20]). Namely, any totally real submanifold $M$ of $C^{n}$ admits local polynomial approximation (Theorem 7.1). Also, if $M$ is a compact totally real submanifold of $\boldsymbol{C}^{n}$ then continuous functions on $M$ can be approximated by polynomials if and only if $M$ is polynomially convex, which was shown some time ago by Helson and Quigley for smooth curves in $C^{n}$ [5].

In $\S 2$ we give a survey of the basic definitions and concepts needed in the paper. In $\$ \S 3,4$, and 5 we develop tools necessary to prove the approximation theorems which we present in $\S 6$. In $\S 3$ we show that a $C^{\infty}$ function $f$ defined on a totally real submanifold $M$ can be extended to a $C^{\infty}$ function $\tilde{f}$ defined near $M$ so that $\bar{\partial} \tilde{f}$ vanishes to high order at $M$. In $\S 4$ we see that totally real submanifolds have arbitrarily small tubular neighborhoods which are strongly pseudoconvex. And in $\S 5$ we bring together some elementary results in partial differential equations along with the statement of an important result from Hörmander's paper concerning the solution to the $\bar{\partial}$-Neumann problem in a pseudoconvex domain with $L^{2}$ estimates for the solution.

Some of the results of this paper were announced in [12]. For real-analytic submanifolds, an independent proof of our principal result (Theorem 6.1) (in its original form) was given in [15], and has been extended to certain classes of realanalytic subvarieties [18]. For 2-manifolds in $C^{n}$ this result was proven earlier in a slightly more general form by M. Freeman in [2], using the techniques of Wermer and Bishop.

At this point the authors would like to express their indebtedness to Lars Hörmander. The basic techniques used here of finding an appropriate extension

${ }^{2}$ ) This was also found independently by C. Stenard (unpublished). 
from a totally real submanifold $M$ of a smooth function $u$ on $M$ so that $\bar{\partial} u$ vanishes to high order on $M$ (the formal Cauchy-Kovalevsky theorem in this situation) and then solving a $\bar{\partial}$-Neumann problem in an appropriate tubular neighborhood was outlined in a letter to the second author in 1965. The original proofs of some of the theorems presented here (as outlined in [12]) were based on this technique along with a detailed analysis of the dependence on the domain of the estimates in [10]. At a later date Hörmander pointed out the applicability of Proposition 5.4 to this problem, enabling us to simplify our analysis considerably, by allowing us to use the $L^{2}$ estimates of [7]. It is this approach which we have carried out here. More recently, Hörmander and Wermer have also used these same techniques to generalize Theorem 6.1 and some theorems of Wermer and Freeman (see [22]), in which they consider totally real submanifolds with "singularities", i.e. points where the holomorphic tangent space is nonzero.

We would also like to express our gratitude to Joe Kohn, Hugo Rossi and John Wermer for their many helpful comments and continual encouragement during our research in these problems. In particular, Theorem 6.1 was conjectured by Rossi in a letter several years ago, and Wermer supplied us with the example following Corollary 7.4 .

2. Preliminaries. Let $\boldsymbol{R}$ and $\boldsymbol{C}$ denote the fields of real and complex numbers, respectively. Suppose $X$ is a complex manifold( $\left(^{3}\right)$, and let $U$ be an open subset of $X$ (see either [4] or [8] as a reference on several complex variables). Let $\mathcal{O}(U), C^{\infty}(U)$, $E_{(p, q)}(U)$ denote the algebras of holomorphic functions, $C^{\infty}$ functions, and $C^{\infty}(p, q)$ forms on $U$, respectively. If $S$ is any subset of $X$, let $\dot{\mathcal{O}}(S), \dot{C}^{\infty}(S), \dot{E}_{(p, q)}(S)$ be the inductive limits of $\mathcal{O}(U), C^{\infty}(U), E_{(p, q)}(U)$, respectively, where $U$ runs over all open sets in $X$ such that $U \supset S$. We shall let $E_{(p, q)}(S)$ be the restriction of $\dot{E}_{(p, q)}(S)$ to $S$. We also set

$$
\begin{aligned}
C_{0}^{\infty}(S) & =\left\{f \in C^{\infty}(S): f \text { has compact support in } S\right\}, \\
D_{(p, q)}(S) & =\left\{f \in E_{(p, q)}(S): f \text { has compact support in } S\right\} .
\end{aligned}
$$

If $K$ is a compact set in $X$, let $C(K)$ denote the Banach algebra of continuous complex-valued functions on $K$ with the maximum norm topology. Let $A(K)$ denote the closure of the natural image of $\mathcal{O}(K)$ in $C(K)$, and if $U$ is open in $X$ with $U \supset K$, let $A(K, U)$ denote the closure of the natural image of $\mathcal{O}(U)$ in $C(K)$.

Suppose $M$ is a real differentiable $C^{1}$ submanifold of the complex manifold $X$ with tangent bundle $T(M)$, which is a real subbundle of $T(X) \mid M$, where $T(X)$ is the tangent bundle to $X$. Let $H_{x}(M)$ be the maximal complex subspace of $T_{x}(X)$ such that $H_{x}(M) \subset T_{x}(M) . H_{x}(M)$ is, by definition, the holomorphic tangent space to $M$ at $x$, and we set $h_{x}(M)=\operatorname{dim}_{C} H_{x}(M)$.

(3) In this paper we assume that all manifolds considered have a countable basis for their topology. 
Proposition 2.1. If $\operatorname{dim}_{R} T_{x}(M)=k$ and $\operatorname{dim}_{C} T_{x}(X)=n$, then

$$
\max (0, k-n) \leqq h_{x}(M) \leqq[k / 2] .
$$

The proof is elementary and is therefore omitted.

Definition 2.2. Suppose $M$ is a connected $C^{1}$ submanifold of a complex manifold $X$.

(a) $M$ is called a CR submanifold of $X$ if $h_{x}(M)$ is constant on $M$, and the constant $h_{x}(M)$ is called the CR dimension of $M$, which we shall denote by CR-dim $M$.

(b) A CR submanifold of CR dimension zero is called a totally real submanifold of $X$.

REMARK 2.3. 1. In view of Proposition 2.1, a totally real submanifold $M$ of a complex manifold $X$ of pure dimension $n$ has necessarily $\operatorname{dim}_{R} M \leqq n$.

2. A real curve or a real hypersurface in $X$ is automatically a CR submanifold.

3. Examples of totally real submanifolds of $C^{n}$ are given by a real submanifold of $\boldsymbol{R}^{n}$, where $\boldsymbol{R}^{n}$ is canonically embedded in $\boldsymbol{C}^{n}$, by the distinguished boundary of the unit polydisc, and by any real curve.

4. It is shown in [17] that any "perturbation" of a totally real embedding of a manifold in $\boldsymbol{C}^{n}$ is still a totally real embedding of the manifold.

Let $M$ be a CR submanifold of a complex manifold $X$; then the tangent bundle to $M$ splits:

$$
T(M)=H(M) \oplus Y(M),
$$

where $H(M)$ is a complex vector bundle over $M$ with fiber $H_{x}(M)$, and $Y(M)$ is isomorphic to $T(M) / H(M)$. Also, $H(M) \otimes_{R} C$ splits into a Whitney sum of subbundles of type $(1,0)$ and $(0,1)$ respectively:

$$
H(M) \otimes_{R} C \cong H(M) \oplus \bar{H}(M) ;
$$

where $\bar{H}_{x}(M)$ is the vector space of conjugates of $H_{x}(M)$. We also have the dual bundles

$$
H^{*}(M) \otimes_{R} C \cong H^{*}(M) \oplus \bar{H}^{*}(M) .
$$

If $E$ is a $C^{\infty}$ vector bundle over a manifold $X$, denote by $\Gamma(S, E)$ the module of $C^{\infty}$ sections of $E$ over $S \subset X$. In particular $\Gamma(H(M))=\Gamma(M, H(M))$ is the module of complex vector fields of type $(1,0), \Gamma\left(\bar{H}^{*}(M)\right)$ is the module of differential forms on $M$ of type $(0,1)$, etc. Note that

$$
\Gamma\left(H^{*}(M)\right) \subset E_{(1,0)}(M), \quad \Gamma\left(\bar{H}^{*}(M)\right) \subset E_{(0,1)}(M),
$$

and that these are the differential forms tangential to $M$ of type $(1,0)$ and $(0,1)$ respectively. If $s_{1}, \ldots, s_{k}$ are sections of $E$ such that $\left\{s_{1}(y), \ldots, s_{k}(y)\right\}$ is a basis for $E_{y}$ for $y$ near $x \in X$, then we say that $\left\{s_{1}, \ldots, s_{k}\right\}$ is a local basis for $E$ at $x$. If, as before, $M$ is a $C^{\infty} \mathrm{CR}$ submanifold of a complex manifold $X$, and $\left\{V_{1}, \ldots, V_{m}\right\}$ is a local basis for $\bar{H}(M)$ at $x \in M$, then $\left\{V_{1}, \ldots, \bar{V}_{m}\right\}$ is a local basis for $\bar{H}(M)$ at $x$. 
Definition 2.4. Let $f \in C^{\infty}(M)$. We say that $f$ is a CR function at $x \in M$ if $\bar{V}_{f} f(y)=0$, for $y$ near $x$ and $j=1, \ldots, m$, that is, if $f$ satisfies the induced CauchyRiemann equations on $M$ near $x \in M$.

This definition is clearly independent of the choice of a local basis for $H(M)$ at $x$. If now $\mathcal{O}_{M}$ denotes the sheaf of germs of CR functions on $M$, for any $K \subset \subset M$ and $U \supset K, U$ open in $M$, we will write $A_{M}(K)\left(A_{M}(K, U)\right)$ for the closure of the natural image of $\Gamma\left(K, \mathcal{O}_{M}\right)\left(\Gamma\left(U, \mathcal{O}_{M}\right)\right)$ in $C(K)$, respectively. Finally, we have the following elementary lemma, whose proof will be omitted. Suppose $\operatorname{dim}_{C} X=n$ and $M$ is a CR submanifold of $X$ of CR dimension $m$.

LEMMA 2.5. If $x \in M$ and $\rho_{1}, \ldots, \rho_{l}$ are real-valued $C^{\infty}$ functions defined on a neighborhood $N$ of $x$ in $X$ such that

(i) $d \rho_{1} \wedge \cdots \wedge d \rho_{l} \neq 0$ on $M \cap N$,

(ii) $M \cap N=\left\{x \in N ; \rho_{1}(x)=\cdots=\rho_{l}(x)=0\right\}$,

then there are $n-m=k$ 's say $\rho_{1}, \ldots, \rho_{k}$ such that

$$
\bar{\partial} \rho_{1} \wedge \cdots \wedge \bar{\partial} \rho_{k} \neq 0 \quad \text { near } x \text { on } M \text {. }
$$

Furthermore, a function $f \in C^{\infty}(M)$ is a CR-function near $x$ if and only if for any $\tilde{f} \in \dot{C}^{\infty}(M)$ such that $\tilde{f} \mid M=f$,

$$
\bar{\partial} \tilde{f} \wedge \bar{\partial} \rho_{1} \wedge \cdots \wedge \bar{\partial} \rho_{k}=0
$$

on $M$ near x.

If $K$ is a subset of $C^{n}$, we shall have occasion to use the distance function to $K$ in $C^{n}$ defined to be

$$
d_{K}(z)=\inf _{x \in K}|z-x|
$$

where $\mid$ is the Euclidean norm in $\boldsymbol{C}^{n}$.

3. Extension of $\mathbf{C R}$ functions from $\mathbf{C R}$ submanifolds. In this section we show that a CR function $f$, defined on a CR submanifold of a complex manifold $X$, can be extended to a function $f$ defined on a neighborhood of $M$ in $X$ in such a way that $\bar{\partial} f$ vanishes to a high order of the local distance to $M$. We treat totally real submanifolds first in complete detail, and then extend the analysis to CR submanifolds with positive $\mathrm{CR}$ dimension.

First we need some notation. We introduce two types of multi-indices as follows: for a positive integer $q$, we set $I=\left(i_{1}, \ldots, i_{N}\right)$ where $i_{j} \in\{1, \ldots, q\}$, and $\|I\|=N$. For $q$ numbers $\rho_{1}, \ldots, \rho_{q}$ we set $\rho_{I}$ equal to the product $\rho_{i_{1}} \cdots \rho_{i_{N}}$, and consequently,

$$
\sum_{\|I\|=N} \rho_{I}=\sum_{i_{1} \ldots i_{N}=1}^{q} \rho_{i_{1}} \cdots \rho_{i_{N}} .
$$

On the other hand, for a $q$-tuple of positive integers $\beta=\left(\beta_{1}, \ldots, \beta_{q}\right)$, we set $|\beta|$ $=\beta_{1}+\cdots+\beta_{q}$, and $\rho^{\beta}=\rho_{1}^{\beta} \cdots \rho_{q}^{\beta} q$. We will use this last notation with $\rho_{i}$ replaced by the operator $D_{x_{i}}, 1 \leqq i \leqq 2 n$, the derivative with respect to $x_{i}$ in the space $\boldsymbol{R}^{2 n}=C^{n}$. 
We state now the following lemma, the proof of which is a variant of the formal part of the proof of Cauchy-Kovalevsky's theorem. (See also Hörmander [8, p. 32] for the case in which $M$ has real codimension 1.)

LEMMA 3.1. Let $M$ be a totally real submanifold of a complex manifold $X$. Suppose $\rho_{1}, \ldots, \rho_{m}$ are real-valued $C^{\infty}$ functions defined on a neighborhood $U$ of $M$ such that

$$
M=\left\{x \in U: \rho_{1}(x)=\cdots=\rho_{m}(x)=0\right\},
$$

and the rank of the Jacobian matrix of the $\rho$ 's in local coordinates is maximal. If $q, r$ are nonnegative integers, then any $f \in C^{\infty}(M)$ is the restriction to $M$ of a function $u$ defined in a neighborhood $W$ of $M$ such that

$$
D^{\alpha} \bar{\partial} u=\sum_{|\beta|=r} h_{\beta} \rho^{\beta} \quad \text { for }|\alpha| \leqq q
$$

where $h_{\beta} \in E_{(0,1)}(W)$.

Proof. Obviously it suffices to show that we can find an extension $u$ and $h_{\beta}$ such that

$$
\bar{\partial} u=\sum_{|\beta|=q+r} h_{\beta} \rho^{\beta} .
$$

The next observation is that there being no holomorphic tangent vectors at any point $p \in M$, the kernel of the matrix whose rows are the $\partial \rho_{i}$ evaluated at $p$, is zero. For any vector $\left(\zeta_{1}, \ldots, \zeta_{n}\right)$ which is annihilated by this matrix is by definition a holomorphic tangent vector at $p$. Therefore, by Fredholm's "alternative" theorem, given any vector $\zeta=\left(\zeta_{1}, \ldots, \zeta_{n}\right)$, there exists another vector $\eta=\left(\eta_{1}, \ldots, \eta_{m}\right)$ such that

$$
\zeta=\sum_{i=1}^{m} \eta_{i} \bar{\partial}_{\rho_{i}}(p)
$$

The $\rho_{i}$ being $C^{\infty}$ functions, we have that $\eta_{i}$ will be $C^{\infty}$ functions if the $\zeta_{i}$ are $C^{\infty}$, and in particular, we conclude that the set $\left\{\bar{\partial}_{\rho_{i}}\right\}, i=1, \ldots, m$, generates the module $\dot{E}_{(0,1)}(M)$ over the ring $\dot{C}^{\infty}\left(M_{\alpha}\right)$.

Now we can pick a locally finite covering of $M$ by open coordinate neighborhoods $W_{\alpha} \subset U$, such that on $W_{\alpha}$, there are $n$ linearly independent forms $\bar{\partial}_{\rho_{i_{1}}}, \ldots, \bar{\partial} \rho_{i_{n}}$ ( $\left.n=\operatorname{dim}_{C} X\right)$ spanning $E_{(0,1)}\left(W_{\alpha}\right)$ over $C^{\infty}\left(W_{\alpha}\right)$. This we can do since near each point of $M$ there are $n$ linearly independent $\bar{\partial} \rho_{j}$ 's. Let $\left\{g_{\alpha}\right\}$ be a $C^{\infty}$ partition of unity subordinate to the covering $\left\{W_{\alpha}\right\}$. Let $\psi$ be any $C^{\infty}$ extension of $f \in C^{\infty}(M)$ to $U$, and set $\psi_{\alpha}=g_{\alpha} \psi$. We will now prove the theorem locally, i.e., that there is an extension $U_{\alpha}$ of $f \mid W_{\alpha} \cap M$ to the whole of $W_{\alpha}$ satisfying (3.1). To that end, take $\bar{\partial} \psi_{\alpha}$ for some fixed $\alpha$, and after reordering the $\rho_{i}$ 's if necessary, suppose $\bar{\partial} \rho_{1}, \ldots, \bar{\partial} \rho_{n}$ is a basis for $E_{(0,1)}\left(W_{\alpha}\right)$ over $C^{\infty}\left(W_{\alpha}\right)$. Then there is a unique way of writing

$$
\bar{\partial} \psi_{\alpha}=\sum_{i=1}^{n} h_{i} \bar{\partial} \rho_{i}, \quad h_{i} \in C_{0}^{\infty}\left(W_{\alpha}\right), \quad i=1, \ldots, n,
$$


(recall that $\psi_{\alpha} \in C_{0}^{\infty}\left(W_{\alpha}\right)$ ), and similarly,

$$
\bar{\partial} h_{i}=\sum_{j=1}^{n} h_{i j} \bar{\partial} \rho_{j}, \quad h_{i j} \in C_{0}^{\infty}\left(W_{\alpha}\right), i, j=1, \ldots, n .
$$

Inductively we can define $h_{I}$ for any multi-index $I$ with entries from 1 to $n$, by $\bar{\partial} h_{J}=\sum_{j=1}^{n} h_{J j} \bar{\partial} \rho_{j}$. It is easy to show by induction that for any $I$ the $h_{I}$ are symmetric, that is, they remain invariant under permutations of the entries of $I$. In fact, suppose the $h_{I}$ 's are symmetric when $\|I\| \leqq N$. For any $J$ with $\|J\|=N-1$, write

$$
\bar{\partial} h_{J}=\sum_{j=1}^{n} h_{J j} \bar{\partial} \rho_{j} .
$$

Then, as $\bar{\partial}^{2}=0$, we have

$$
\sum_{j=1}^{n} \bar{\partial} h_{j j} \wedge \bar{\partial} \rho_{j}=\sum_{j=1}^{n} \sum_{k=1}^{n} h_{j j k} \bar{\partial} \rho_{k} \wedge \bar{\partial} \rho_{j}=0,
$$

and therefore,

$$
\sum_{k<j}\left(h_{J j k}-h_{J k j}\right) \bar{\partial} \rho_{k} \wedge \bar{\partial} \rho_{j}=0 .
$$

But the $(0,2)$ forms $\bar{\partial} \rho_{k} \wedge \bar{\partial} \rho_{j}, k<j, k, j=1, \ldots, n$, are linearly independent over $C^{\infty}\left(W_{\alpha}\right)$, which implies that

$$
h_{J j k}=h_{J k j} .
$$

It only remains to show that $h_{J j k}$ remains invariant under a permutation leaving at least one of the last two subindices fixed, but this is obvious from (3.4), our inductive hypothesis, and the fact that the expansion (3.3) is unique.

If now $N=q+r$ as in (3.1), the desired function $u_{\alpha}$ defined on $W_{\alpha}$ is

$$
u_{\alpha}=\psi_{\alpha}+\sum_{m=1} \frac{(-1)^{m}}{m !} \sum_{\|I\|=m} h_{I} \rho_{I} .
$$

To see this, observe first that $u_{\alpha}\left|M \cap W_{\alpha}=\psi_{\alpha}\right| M \cap W_{\alpha}=f \mid M \cap W_{\alpha}$, for all the $\rho$ 's vanish on $M$. Secondly, because of the symmetry of the $h_{I}$, it follows easily that

$$
\bar{\partial} u_{\alpha}=\frac{(-1)^{N}}{N !} \sum_{\|I\|=N}\left(\bar{\partial} h_{I}\right) \rho_{I},
$$

which proves the lemma locally.

Finally to conclude with the proof of the lemma, we carry out the same construction on each of the $W_{\alpha}$, and define $u=\sum_{\alpha} u_{\alpha} . u$ is defined on $W=\bigcup_{\alpha} W_{\alpha} \supset M$, furthermore $u \mid M=f$, and

$$
\bar{\partial} u=\sum_{\alpha} \bar{\partial} u_{\alpha}=\sum_{\alpha} \sum_{\|I I\|=N} h_{I} \rho_{I},
$$

whereby, after a change in notation we get

$$
\bar{\partial} u=\sum_{|\beta|=N} \tilde{h}_{\beta} \rho^{\beta} .
$$


Corollary 3.2. Assume $M$ is as in Lemma 3.1 and $X=C^{n}$. For any $f \in C^{\infty}(M)$ and any $m>0$, there is a $C^{\infty}$ function $U$ defined on a neighborhood of $M$ such that

$$
D^{\alpha} \bar{\partial} u=O\left(d_{M}^{r}\right), \quad|\alpha| \leqq q ; \quad u \mid M=f
$$

where $d_{M}$ is given by (2.1).

This proof is straightforward and is left to the reader.

We will now prove a similar extension lemma for CR functions on a CR manifold of positive CR dimension, but here the result will be a local one; we cannot use a partition of unity as above for multiplication of a CR function by a function of compact support does not yield a CR function.

We introduce first some additional notation. Let $M$ be a $C^{\infty}$ submanifold of some open set in $C^{n}$, and suppose, for simplicity that $0 \in M$. Then $\pi_{T}$ will denote the projection $\pi_{T}: C^{n} \rightarrow T_{0}(M)$, where we have identified $T_{0}\left(C^{n}\right)$ with $C^{n}$. Also $g$ will be the canonical local diffeomorphism from $T_{0}(M)$ to $M$, given by the orthogonal projection of $M$ into $T_{0}(M)$, which is well defined near 0 . Let $N_{0}(M)$ denote the orthogonal complement to $T_{0}(M)$ at 0 , and let $\pi_{N}$ be the orthogonal projection of $C^{n}$ onto $N_{0}(M)$. Then, if $B_{T}(\varepsilon)$ is the open ball of radius $\varepsilon$ about 0 in $T_{0}(M)$ and $B_{N}(\varepsilon)$ the corresponding ball in $N_{0}(M)$, we set

$$
W_{T}(\varepsilon)=\pi_{T}^{-1}\left(B_{T}(\varepsilon)\right), \quad W_{N}(\eta)=\pi_{N}^{-1}\left(B_{N}(\eta)\right)
$$

for $\varepsilon$ and $\eta \geqq 0$, and $U(\varepsilon, \eta)=W_{T}(\varepsilon) \cap W_{N}(\eta)$. We note that $U(\varepsilon, \eta)$ is a convex neighborhood of 0 , hence Stein, and it is clear that for small $\varepsilon, g\left(B_{T}(\varepsilon)\right)=W_{T}(\varepsilon) \cap M$, and that $\left\{W_{T}(\varepsilon) \cap M\right\}$ is a fundamental system of neighborhoods of 0 in $M$. Now we can state the following

LEMMA 3.3. Let $M$ be a $\mathrm{CR}$ submanifold of an open set in $C^{n}$, and let $0 \in M$. There exists $\varepsilon_{0}, \eta_{0}>0$ such that $U=U\left(\varepsilon_{0}, \eta_{0}\right) \supset M \cap W_{T}\left(\varepsilon_{0}\right)$ and furthermore, if $f$ is a $\mathrm{CR}$ function on $U\left(\varepsilon, \eta_{0}\right) \cap M$, where $0<\varepsilon \leqq \varepsilon_{0}$, and $q$ and $r$ are positive integers, then there is a function $u \in C^{\infty}\left(U\left(\varepsilon, \eta_{0}\right)\right)$ such that

(a) $u \mid U\left(\varepsilon, \eta_{0}\right) \cap M=f$,

(b) $D^{\alpha}(\bar{\partial} u)=O\left(d_{M}^{r}\right)$, for $|\alpha| \leqq q$,

where, as before, $d_{M}$ (given by (2.1)) is the distance from a point in $U\left(\varepsilon, \eta_{0}\right)$ to $U\left(\varepsilon, \eta_{0}\right) \cap M$.

Proof. As in Lemma 3.1 and its corollary, it suffices to show that for any positive integer $N$ we can find an extension $u$ of $f$ satisfying $\bar{\partial} u=\sum_{|\beta|=N} h_{\beta} \rho^{\beta}$, where $\rho=\left(\rho_{1}, \ldots, \rho_{m}\right)$ is an $m$-tuple of $C^{\infty}$ real-valued functions such that

$$
M \cap U\left(\varepsilon_{0}, \eta_{0}\right)=\left\{z \in U\left(\varepsilon_{0}, \eta_{0}\right) ; \rho_{1}(z)=\cdots=\rho_{m}(z)=0\right\},
$$

and such that the Jacobian matrix of the $\rho$ 's has rank $m$ on $M \cap U\left(\varepsilon_{0}, \eta_{0}\right)$ for some $\varepsilon_{0}, \eta_{0}$. Let us first define the functions $\rho$. By an affine unitary change of coordinates we can assume that $t=\left\{t_{1}, t_{2}, \ldots, t_{m}\right\}$ is a set of real coordinates in $N_{0}(M)$, and $u=\left\{u_{1}, \ldots, u_{2 n-m}\right\}$ is a set of real coordinates for $T_{0}(M)$. Then, in a neighborhood 
$V$ of 0 , we can represent $M$ as $t=\varphi(u)$, where the vector-valued function $\varphi$ vanishes to second-order at $u=0$. We set $\rho_{i}=t_{i}-\varphi_{i}(u), i=1, \ldots, m$. Next assume (by reordering the $\rho$ 's, if necessary) that $\left\{\bar{\partial}_{\rho_{1}}, \ldots, \bar{\partial} \rho_{k}\right\}$ is a maximal subset of $\left\{\bar{\partial} \rho_{1}, \ldots, \bar{\partial} \rho_{m}\right\}$ of linearly independent vectors at 0 . Then this set will still be linearly independent on some neighborhood of $0, V^{\prime} \subset V$. Now we choose $\varepsilon_{0}, \eta_{0}$ in such a way that

$$
M \cap W_{T}\left(\varepsilon_{0}\right) \subset U\left(\varepsilon_{0}, \eta_{0}\right) \subset V^{\prime} .
$$

Let $U=U\left(\varepsilon_{0}, \eta_{0}\right)$. For a real vector $\tau=\left(\tau_{1}, \ldots, \tau_{m}\right)$ we define the $\tau$-translate $M_{\tau}$ of $M$ as

$$
M_{\tau}=\left\{z \in V ; \rho_{i}(z)=\tau_{i}, i=1, \ldots, m\right\},
$$

and we observe that $U$ is contained in a union of disjoint translates of $M \cap U$. Therefore, if we are given a $C R$ function $f$ on $M \cap U$, we can extend it by translation to a function $u_{0}$ defined on $U$ which is independent of the variables $t_{1}, \ldots, t_{m}$. Now, saying that $f$ is CR on $M \cap U$ is the same as saying that for any smooth extension $u$ of $f$, (Lemma 2.5):

$$
\bar{\partial} u \wedge \bar{\partial} \rho_{1} \wedge \cdots \wedge \bar{\partial} \rho_{k}=0 \text { on } M \cap U .
$$

In particular

$$
\bar{\partial} u_{0} \wedge \bar{\partial} \rho_{1} \wedge \cdots \wedge \bar{\partial} \rho_{k}=0 \text { on } M \cap U .
$$

But the components of the vectors $\bar{\partial} u_{0}, \bar{\partial} \rho_{1}, \ldots, \bar{\partial} \rho_{k}$ are all independent of the variables $t_{1}, t_{2}, \ldots, t_{m}$, and therefore the equality (3.6) holds throughout $U$, that is to say,

$$
\bar{\partial} u_{0}=\sum_{i=1}^{k} h_{i} \bar{\partial} \rho_{i} \quad \text { on } U
$$

with $h_{i} \in C^{\infty}(U)$. From (3.7) the proof proceeds along the same lines as that of Lemma 3.1 , by observing that

$$
0=\bar{\partial}^{2} u_{0}=\sum_{i=1}^{k} \bar{\partial} h_{i} \wedge \bar{\partial} \rho_{i} \text { on } U
$$

and therefore $\bar{\partial} h_{i} \wedge \bar{\partial} \rho_{1} \wedge \cdots \wedge \bar{\partial} \rho_{k}=0$ on $U$ for $i=1, \ldots, k$, which allows us to write on $U$

$$
\bar{\partial} h_{i}=\sum_{j=1}^{k} h_{\imath j} \bar{\partial} \rho_{j}
$$

and so on. We omit further details. Q.E.D.

REMARK 3.4. If $M$ is a real hypersurface, the result is true globally (Hörmander, loc. cit.). We do not know whether a global result is true for lower dimensional CR manifolds.

REMARK 3.5 (ADDED IN PROOF). An oral communication from M. Freeman indicates that a global version of the above extension theorem has been proved 
for lower dimensional $\mathrm{CR}$ submanifolds of minimal $\mathrm{CR}$ dimension (the generic case).

4. Tubular neighborhoods of totally real submanifolds. Let $M$ be a $C^{\infty}$ submanifold of $C^{n}$, and let $\nu$ be the normal bundle to $M$ with respect to the usual Hermitian metric in $C^{n}$. Then the zero cross section in $\nu$ can be identified with $M$, and it is well known that there is a neighborhood of the zero cross section in $\nu$ which is diffeomorphic to a neighborhood of $M$ in $C^{n}$, which is called a tubular neighborhood of $M$ (see [11, p. 57]). If $M$ is compact, then we let $\nu(\varepsilon)$ be the bundle of vectors of length $<\varepsilon$, and we have, for sufficiently small $\varepsilon, \nu(\varepsilon)$ is diffeomorphic to $T(\varepsilon)=\left\{z \in C^{n}: d_{M}(z)<\varepsilon\right\}$, which is called the tubular neighborhood of $M$ of radius $\varepsilon$. We have the following proposition.

Proposition 4.1. Let $M$ be a totally real submanifold of $C^{n}$, and let $\varphi=d_{M}^{2}$, then at any point $p \in M$ the complex Hessian of $\varphi$,

$$
\frac{\partial^{2} \varphi}{\partial z_{i} \partial \bar{z}_{j}}(p)
$$

is a positive definite matrix, and hence $\varphi$ is strictly plurisubharmonic in a neighborhood of $x$. Moreover, $d \varphi(p)=0$.

Proof. Since $M$ is totally real, it follows from elementary linear algebra that we can make a holomorphic linear change of coordinates in $C^{n}$ so that the affine tangent space to $M$ at $p$ is given by

$$
T_{p}(M)=\left\{\left(z_{1}, z_{2}, \ldots, z_{n}\right) \in C^{n}, y_{1}=\cdots=y_{k}=z_{k+1}=\cdots=z_{n}=0\right\},
$$

where $z_{j}=x_{j}+i y_{j}, j=1, \ldots, n, p=(0, \ldots, 0)$, and $\operatorname{dim}_{R} M=k$. Now let $\delta(z)$ be the normal distance from the point $z$ in $C^{n}$ to the affine subspace $T_{p}(M)$. It is easy to see that $d_{M}(z)=\delta(z)+O\left(|z|^{2}\right)$, and moreover we have explicitly

$$
\delta^{2}(z)=\sum_{i=1}^{k} y_{i}^{2}+\sum_{i=k+1}^{n}\left|z_{i}\right|^{2},
$$

which has a positive definite complex Hessian in $\boldsymbol{C}^{n}$.

Then we have

$$
\varphi(z)=\delta^{2}(z)+O\left(|z|^{3}\right)
$$

which therefore has a positive definite complex Hessian in some neighborhood of $(0, \ldots, 0)=p$. Q.E.D.

COROLlaRY 4.2. Let $M$ be a compact totally real submanifold of $\boldsymbol{C}^{n}$, then for sufficiently small $\varepsilon, T(\varepsilon)$ is a strongly pseudoconvex neighborhood of $M$.

We want to generalize this type of situation to submanifolds with boundary, and for this we need a definition. 
Definition 4.3. A finite totally real submanifold $M$ in a complex manifold $X$ is the closure of a relatively compact connected open set (subdomain) $M^{0} \subset \subset M^{\prime}$, where $M^{\prime}$ is a totally real submanifold of $X$, and $M^{0}$ has a $C^{\infty}$ boundary $\partial M^{0}=\partial M$.

Note that a finite totally real submanifold is by definition compact in $X$. We shall first globalize Proposition 4.1 and then generalize Corollary 4.2 to finite totally real submanifolds.

Proposition 4.4. Let $M$ be a totally real submanifold of a complex manifold $X$. Then there is a strictly plurisubharmonic function $\varphi$ defined in a neighborhood $U$ of $M$ such that $M=\{x \in U ; \varphi(x)=0\}$.

Proof (cf. [15]). By virtue of Proposition 4.1 we can find an open countable cover $\left\{U_{j}\right\}$ of $M$ and strongly plurisubharmonic functions $\varphi_{j}$ in $U_{j}$ such that

$$
M \cap U_{j}=\left\{x \in U_{j}: \varphi_{j}(x)=0\right\}, \quad d \varphi_{j} \mid M \cap U_{j}=0 .
$$

Let $\left\{\alpha_{j}\right\}$ be a partition of unity subordinate to $\left\{U_{j}\right\}$ with $\alpha_{j} \geqq 0$, and set $\varphi=\sum_{j} \alpha_{j} \varphi_{j}$. It follows easily from (4.1) that $\varphi$ is defined in $V=\bigcup_{j} U_{j}$, is strictly plurisubharmonic at each point of $M$, and that $M=\{x \in V: \varphi(x)=0\}$. Thus $\varphi$ is strictly plurisubharmonic in some neighborhood of $M$ and the proposition is proven. Q.E.D.

Proposition 4.5. Let $M$ be a finite totally real submanifold of a complex manifold $X$. Then there is a neighborhood $U$ of $M$ in $X$ and a strictly plurisubharmonic function $\varphi$ defined in $U$ such that $M=\{x \in U: \varphi(x)=0\}$.

Proof. Suppose $M$ is given as in Definition 4.3, where $M^{\prime}$ is a totally real submanifold of $X$. Then let $\varphi^{\prime}$ be the strictly plurisubharmonic function for $M^{\prime}$ given by Proposition 4.4. Find a function $\psi \in C^{\infty}(X)$ such that $\psi$ vanishes on $M$, and $\psi>0$ on $X-M$. This is easily done, since $M$ has a smooth boundary. Then set $\varphi=\varphi^{\prime}+\varepsilon \psi, \varepsilon \in \boldsymbol{R}$. We can choose $\varepsilon$ small enough so that $\varphi$ is strictly plurisubharmonic on $M$ which is compact in $M^{\prime}$. Q.E.D.

THEOREM 4.6. Let $M$ be any finite or compact totally real submanifold of a complex manifold $X$, then there is a Stein neighborhood $U$ of $M$ and a proper holomorphic embedding of $U$ into $C^{n}$ for some $N$ so that $M$ becomes a finite or compact totally real submanifold of complex Euclidean space $C^{N}$.

Proof. This is immediate in view of the preceding propositions, Grauert's solution to the Levi problem, and the Bishop proper holomorphic embedding theorem for Stein manifolds (see [4] or [8]). Q.E.D.

This theorem allows us to study totally real submanifolds in $C^{n}$ without losing any generality, and this is desirable, since then the geometry simplifies even more, as we see in the following theorem.

THEOREM 4.7. Let $M$ be a finite or compact totally real submanifold of $\boldsymbol{C}^{n}$, then for sufficiently small $\varepsilon, T(\varepsilon)=\left\{x \in C^{n}: d_{M}(x)<\varepsilon\right\}$ is a pseudoconvex domain in $C^{n}$. 
Proof. In the compact case, we have that $T(\varepsilon)$ is a strongly pseudoconvex domain for small $\varepsilon$, by Corollary 4.2. So suppose $M$ is a finite submanifold of $C^{n}$ and $M^{\prime}$ is given by Definition 4.3. Let $\varphi=d_{M}^{2}, \varphi^{\prime}=d_{M^{\prime}}^{2}$, and $\varphi^{\prime \prime}=d_{\partial M}^{2}$. Then set

$$
\begin{aligned}
W(\varepsilon) & =\left\{x \in C^{n}: \varphi(x)<\varepsilon\right\}, \quad W^{\prime}(\varepsilon)=\left\{x \in C^{n}: \varphi^{\prime}(x)<\varepsilon\right\}, \\
W^{\prime \prime}(\varepsilon) & =\left\{x \in C^{n}: \varphi^{\prime \prime}(x)<\varepsilon\right\}, \quad W(\varepsilon, \eta)=W^{\prime}(\varepsilon) \cap W(\eta) .
\end{aligned}
$$

We have the following lemma which proves the theorem.

LEMMA 4.8. There exists an $\varepsilon_{0}>0$ so that

(1) For $0<\varepsilon \leqq \varepsilon_{0}, T(\sqrt{ } \varepsilon)=W(\varepsilon, \varepsilon)$ is pseudoconvex.

(2) For $0<\eta<\varepsilon \leqq \varepsilon_{0}, W(\varepsilon, \eta)$ is pseudoconvex.

Proof. Since the increasing union of pseudoconvex domains is pseudoconvex, we see that (2) implies (1). Therefore we shall prove (2). Note first that $\varphi^{\prime}$ and $\varphi^{\prime \prime}$ are strictly plurisubharmonic in a neighborhood of $M$ and $\partial M$ respectively, since $M$ is compact in $M^{\prime}$ and $\partial M$ is a compact totally real submanifold of $C^{n}$. Choose $\varepsilon_{0}$ small enough so that for $\varepsilon$ and $\eta \leqq \varepsilon_{0}$ we have that $W^{\prime}(\varepsilon)$ and $W^{\prime \prime}(\eta)$ are strongly pseudoconvex domains. Then for $x_{0} \in \partial W(\varepsilon, \eta)$, where we assume $0<\eta<\varepsilon<\varepsilon_{0}$, we have either

(i) $\varphi\left(x_{0}\right)=\varepsilon, \varphi^{\prime}(x) \leqq \eta$ or

(ii) $\varphi\left(x_{0}\right) \leqq \varepsilon, \varphi^{\prime}(x)=\eta$.

In case (i) it is clear that for $x$ near $x_{0}$ we have $\varphi(x)=\varphi^{\prime \prime}(x)$ since $\eta<\varepsilon$. But then there is a neighborhood $U$ of $x_{0}$ such that $\varphi^{\prime}$ and $\varphi^{\prime \prime}$ are strictly plurisubharmonic in $U$ and

$$
W(\varepsilon, \eta) \cap U=\left\{x \in U: \varphi^{\prime}(x)<\eta, \varphi^{\prime \prime}(x)<\varepsilon\right\},
$$

and thus $W(\varepsilon, \eta)$ is pseudoconvex at $x_{0}$. In case (ii) there is a neighborhood $U$ of $x_{0}$ such that $\varphi^{\prime}$ is strictly plurisubharmonic in $U$ and $W(\varepsilon, \eta)=\left\{x \in U \cdot \varphi^{\prime}(x)<\eta\right\}$. Q.E.D.

COROLlaRY 4.9. Any compact or finite totally real submanifold $M$ of a complex manifold $X$ is $O(U)$-convex with respect to some Stein neighborhood $U$ of $M$ in $X$.

5. $L^{2}$ estimates and the $\bar{\partial}$-Neumann problem. In this section we shall give various results about partial differential equations which will be needed in the following section. All of these results exist in the literature, but we collect them here as a convenient reference for later use. To simplify the presentation we first introduce some standard function spaces and norms.

Let $\Omega$ be open in $\boldsymbol{R}^{m}$, and define, for $u \in C_{0}^{\infty}(\Omega)$, and $s$ a nonnegative integer,

$$
\begin{aligned}
|u|_{\Omega, s} & =\sup _{x \in \Omega ;|\alpha| \leqq s}\left|D^{\alpha} u(x)\right|, \\
\|u\|_{\Omega, s} & =\left\{\sum_{|\alpha| \leqq s} \int_{\Omega}\left|D^{\alpha} u(x)\right|^{2} d x\right\}^{1 / 2} .
\end{aligned}
$$


Let $W_{s}(\Omega)$ denote the completion of $C_{0}^{\infty}(\Omega)$ with respect to the Sobolev norms \|\|$_{\Omega, s}$. Then consider a compact set $K \subset \boldsymbol{R}^{m}$, and for any $u \in C^{k}\left(\boldsymbol{R}^{m}\right)$, define the norm

$$
|u|_{K, k}=\sup _{x \in K ;|\alpha| \leqq k}\left|D^{\alpha} u(x)\right|,
$$

and let $C^{k}(K)$ be the completion of $C^{k}\left(\boldsymbol{R}^{m}\right) \mid K$ with respect to this norm. Of course, $C^{0}(K)$ is the same as the Banach algebra $C(K)$ introduced earlier.

If $K$ is compact in $U$, where $U$ is open in $\boldsymbol{R}^{m}$, then let $d(K, \partial U)$ be the distance from $K$ to $\partial U$ defined by

$$
d(K, \partial U)=\inf _{y \in \partial U}\left(\inf _{x \in K}|x-y|\right)
$$

We shall need the following elementary lemma the proof of which is omitted, being an easy application of a standard partition of unity argument.

Lemma 5.1. Let $U$ and $V$ be two domains in $\boldsymbol{R}^{m}$ contained in a fixed ball $B$ of radius $R$. Suppose that $V \subset \subset U \subset \subset B$, and set $\eta=d(\bar{V}, \partial U)$. Then there exists a function $\varphi \in C_{0}^{\infty}(U)$ such that $\varphi=1$ on a neighborhood of $\bar{V}$ and such that

$$
|\varphi|_{U, s} \leqq C \eta^{-(s+m)}
$$

where $C$ is a constant depending only on $R$ and $m$.

We now have Sobolev's lemma which we shall state in a form suitable for our purposes. First we have the following version in $\boldsymbol{R}^{\boldsymbol{m}}$.

Proposition 5.2 (Sobolev). Suppose $u \in W_{s}\left(\boldsymbol{R}^{m}\right)$, where $s>[m / 2]+k$, then $u \in C^{k}\left(\boldsymbol{R}^{m}\right)$, and moreover there exists a constant $C$, depending only on $s$ and $m$, such that

$$
|u|_{R^{m}, k} \leqq C\|u\|_{R^{m}, s} .
$$

This is easily proved by means of Fourier transforms (see [6, p. 40]). We now want to modify this (in a well-known manner) to obtain estimates for bounded domains.

Proposition 5.3. Let $\Omega$ be a bounded domain in $\boldsymbol{R}^{m}$, contained in some fixed ball of radius $R$, and suppose $K$ is a compact subset of $\Omega$ with $\eta=d(K, \partial \Omega)$. Then for any $k \geqq 0$, and for any $s>[m / 2]+k$, there exists a constant $C$, depending only on $R, m$ and $s$, such that for any $u \in W_{s}(\Omega)$,

$$
|u|_{K, k} \leqq C \eta^{-(s+m)}\|u\|_{\Omega, s}
$$

This is a straightforward consequence of Lemma 5.1 and Proposition 5.2.

Next we have a special case of a general "elliptic estimate", which can be found in $[8$, p. 140], but we give its proof here in order to obtain an explicit dependence on the constants, as in (5.4). 
Proposition 5.4. Let $\Omega$ be a bounded domain in $C^{n}$, where $\Omega$ is contained in a ball of radius $R$. Suppose $D \subset \subset \Omega$, with $\eta=d(\bar{D}, \partial \Omega)$. Then for each integer $s \geqq 0$, there is a constant $C>0$, depending only on $R, s$, and $n$ such that

$$
\|u\|_{D, s}^{2} \leqq C \eta^{-(s+2 n)}\left(\sum_{j=1}^{n}\left\|\frac{\partial u}{\partial \bar{z}_{j}}\right\|_{\Omega, s-1}^{2}+\|u\|_{\Omega, 0}\right),
$$

for every $u \in C^{\infty}(\bar{\Omega})$.

Proof. Let $\varphi \in C_{0}^{\infty}(\Omega)$ be chosen as in Lemma 5.1 so that $\varphi \equiv 1$ near $\bar{D}$ and (5.1) holds. First we assume $s=1$. Then integration by parts shows that for $u \in C^{\infty}(\bar{\Omega})$,

$$
\int_{\Omega}\left|\frac{\partial(\varphi u)}{\partial z_{j}}\right|^{2} d V=\int_{\Omega}\left|\frac{\partial(\varphi u)}{\partial \bar{z}_{j}}\right|^{2} d V
$$

Therefore, it follows easily that

$$
\int_{D}\left|\frac{\partial u}{\partial z_{j}}\right|^{2} d V \leqq|\varphi|_{\Omega, 1}\left(\left\|\frac{\partial u}{\partial \bar{z}_{k}}\right\|_{\Omega, 0}^{2}+\|u\|_{\Omega, 0}^{2}\right) .
$$

It is then clear that, by using (5.1), we obtain (5.5) for $s=1$. For $s>1$, we proceed by induction, replacing $u$ by $D^{\alpha} u$, and using the same argument. Q.E.D.

We now have the following important result due to Hörmander.

THEOREM 5.5 (HöRMANDER). Let $\Omega$ be a bounded pseudoconvex domain contained in a ball of radius $R$ in $C^{n}$. There exists a constant $C$, depending only on $R$ and $n$, such that for any $f \in E_{(0,1)}(\bar{\Omega})$ with $\bar{\partial} f=0$ in $\Omega$, there exists $a u \in C^{\infty}(\Omega)$ such that $\bar{\partial} u=f$ and $\|u\|_{\Omega, 0} \leqq C\|f\|_{\Omega, 0}$.

This theorem follows easily from Theorem 2.2.3 in [7] (the existence and $L^{2}$ estimate), and from Theorem 4.2.5 in [8] (the regularity).

6. Approximation theorems on $C^{\infty}$ submanifolds. In this section we shall prove various approximation theorems.

THEOREM 6.1. Let $M$ be a compact or finite totally real submanifold of a complex manifold $X$. Then there is an open Stein manifold $U \subset X$ such that $M \subset U$ and the restriction map $\mathcal{O}(U) \rightarrow C^{\infty}(M)$ is dense in the $C^{\infty}$ topology.

Proof. It is clear from Theorem 4.6 that we can take $X=C^{n}$. By Proposition 4.5 there is a strictly plurisubharmonic function $\varphi$ defined in a neighborhood $V$ of $M$ in $X$ such that $M=\{x \in V: \varphi(x)=0\}$. If we set $V_{\varepsilon}=\{z \in V: \varphi(z)<\varepsilon\}$, then for $\varepsilon>0$ small enough, the solution to the Levi problem implies that $V_{\varepsilon}$ is a Stein manifold and a theorem of Bremermann asserts that the restriction map $\mathcal{O}\left(V_{\varepsilon_{1}}\right) \rightarrow \mathcal{O}\left(V_{\varepsilon_{2}}\right)$, $\varepsilon_{1}>\varepsilon_{2}$ is dense in the usual topology of uniform convergence on compact subsets (see [1]). Thus if the map

$$
\mathcal{O}(M) \rightarrow C^{\infty}(M)
$$

is dense, then so is the map $\mathcal{O}\left(N_{\varepsilon_{0}}\right) \rightarrow C^{\infty}(M)$ for some $\varepsilon_{0}>0$, which would prove the theorem. 
To prove (6.1) is dense, we consider first the compact case. Suppose $f \in C^{\infty}(M)$, then for any $k \geqq 0$, and any $\varepsilon>0$, we must find an $h \in \mathcal{O}(M)$ such that $|h-f|_{M, k}<\varepsilon$. Let $N$ be a positive integer, to be determined later, and let $u$ be an extension of $f$ to a neighborhood $W$ of $M$ such that $u \in C^{\infty}(W), u \mid M=f$, and $|\bar{\partial} u(z)| \leqq C d_{M}(z)^{N}$, as given by Corollary 3.2. Letting $T(\varepsilon)=\left\{z \in C^{n}: d_{M}^{2}(z)<\varepsilon\right\}$, as before, and supposing $\varepsilon$ is small enough so that $T(\varepsilon) \subset \subset W$, we use Theorem 5.5 to find a function $v_{\varepsilon} \in C^{\infty}(T(2 \varepsilon))$ so that

$$
\begin{aligned}
\bar{\partial} v_{\varepsilon} & =\bar{\partial} u . \\
\left\|v_{\varepsilon}\right\|_{T(2 \varepsilon), 0} & \leqq C\|\bar{\partial} u\|_{T(2 \varepsilon), 0} .
\end{aligned}
$$

By Sobolev's lemma (Proposition 5.3), we have for $s=n+k+1$, (letting $C$ denote large constants depending only on $\operatorname{diam} M, n$, and $k$ )

$$
\left|v_{\varepsilon}\right|_{M, k} \leqq C \varepsilon^{-(s+2 n)}\left\|v_{\varepsilon}\right\|_{T(\varepsilon), s}
$$

Then we have, from (5.5) and (6.2),

$$
\left\|v_{\varepsilon}\right\|_{T(\varepsilon), s}^{2} \leqq C \varepsilon^{-(s+2 n)}\left(\|\bar{\partial} u\|_{T(2 \varepsilon), s-1}^{2}+\left\|v_{\varepsilon}\right\|_{T(2 \varepsilon), 0}^{2}\right) .
$$

Combining (6.3), (6.4), and (6.5) we obtain

$$
\left|v_{\varepsilon}\right|_{M, k} \leqq C \varepsilon^{-2(s+2 n)}\|\bar{\partial} u\|_{T(2 \varepsilon), s-1} .
$$

We now choose $N$ to be larger than $3 s+4 n-1$. Then we have on $T(2 \varepsilon)$,

$$
\left|D^{\alpha} \bar{\partial} u(z)\right| \leqq C d_{M}^{N-s+1}(z) \leqq C \varepsilon^{N-s+1}, \quad|\alpha| \leqq s-1
$$

which implies that, letting $V(\varepsilon)$ be the volume of $T(2 \varepsilon)$,

$$
\|\bar{\partial} u\|_{T(2 \varepsilon), s-1} \leqq C \varepsilon^{n-s+1} V(\varepsilon),
$$

and thus $\left|v_{\varepsilon}\right|_{M, k} \leqq C \varepsilon V(\varepsilon)$, which tends to zero as $\varepsilon \rightarrow 0$. Set $h=u-v_{\varepsilon}$, and we have $\bar{\partial} h=\bar{\partial} u-\bar{\partial} v_{\varepsilon}=0$ on $T(2 \varepsilon)$ and thus $h \in \mathcal{O}(T(2 \varepsilon))$, and $|h-f|_{M, k}=|h-u|_{M, k}=\left|-v_{\varepsilon}\right|_{M, k}$, which can be made arbitrarily small by taking $\varepsilon$ small enough.

In case $M$ is a finite totally real submanifold, the same analysis applies with one modification. Suppose $M^{\prime}$ is as in Definition 4.3, and $f \in C^{\infty}(M)$ is given. Then extend $f$ to some $f^{\prime} \in C^{\infty}\left(M^{\prime}\right)$ and by Corollary 3.2 applied to $f^{\prime}$ on $M^{\prime}$ we can extend $f$ to some neighborhood $W$ of $M^{\prime}$ so that, if we call $u$ the extension again, we have $\left|D^{\alpha} \bar{\partial} u(z)\right|=O\left(d_{M^{\prime}}^{N}(z)\right)$ for any $N$ given beforehand. Letting $T(\varepsilon)=\left\{z \in C^{n}: d_{M}^{2}(z)<\varepsilon\right\}$ as in the above compact case, we proceed as above, using the extension $u$. Then we note that $d_{M^{\prime}}(z) \leqq d_{M}(z)$ and we have

$$
\left|D^{\alpha} \bar{\partial} u(z)\right| \leqq C d_{M^{\prime}}^{N-s+1}(z) \leqq C d_{M}^{N-s+1}(z)
$$

and proceed as before. Q.E.D.

COROLLARY 6.2. Let $M$ be a compact or finite totally real submanifold of a complex manifold $X$. Then there is an open Stein neighborhood $U$ of $M$ in $X$ such that $A(M, U)=A(M)=C(M)$, i.e. the maps

$$
\mathcal{O}(U) \rightarrow \mathcal{O}(M) \rightarrow C(M)
$$

are dense in the $C(M)$ topology.

A special case of the above is the following (cf. [19], [13]). 
COROllary 6.3. Let $\Gamma$ be a finite or compact $C^{\infty}$ curve in a complex manifold $X$, then there is an open Stein neighborhood $U$ of $\Gamma$ such that $O(U) \rightarrow C^{\infty}(\Gamma)$ is dense, and $A(U, \Gamma)=A(\Gamma)=C(\Gamma)$.

We would like to give an example to show that the hypothesis that $M$ be totally real is essential in the above theorem. Let

$$
D=\left\{z \in C^{2}:\left|z_{1}\right|^{2}+\left|z_{2}\right|^{2}=1, \operatorname{Im} z_{2}=0\right\},
$$

and let $M=\partial D$, a 2 -sphere embedded in $C^{2}$. Then first we note that $\operatorname{dim}_{C} H_{x}(M)=1$ at precisely two points of $M$ and is 0-dimensional otherwise. Secondly it follows easily from a classical Kontinuitätssatz argument that $\mathcal{O}(D) \cong \mathcal{O}(M)$, since $D$ is a 1-parameter family of analytic discs each of whose boundaries lie in $M$, and these analytic discs shrink to a point (see e.g. [14, Theorem 1]). Let $S(A(D)), S(A(M))$ be the spectra of these function algebras (see [4, p. 56]) and then we obtain, since $D$ is convex, $D=S(A(D))=S(A(M))$, and thus $S(A(M)) \neq S(C(M))=M_{0}$. Consequently $A(M) \neq C(M)$.

We also remark that not every compact $n$-manifold $M$ admits a totally real embedding in $C^{n}$, as there are topological obstructions. For instance, if $M$ is orientable then a necessary condition that it admit a totally real embedding in $\boldsymbol{C}^{n}$ is that the Euler characteristic of $M$ vanishes (see [17]).

Let $D$ be a relatively compact subdomain of $C^{n}$, and let $\partial D$ be its boundary. An old unsolved problem is to determine which domains $D$ have the property that

$$
\mathcal{O}(\bar{D}) \rightarrow \mathcal{O}(D) \cap C^{0}(\bar{D})
$$

is a dense map, the topology being uniform convergence on $\bar{D}$. It is easy to see that not all domains in $\boldsymbol{C}^{n}$ have this property, as the classical example

$$
D=\left\{(z, w) \in C^{2}:|\omega|<|z|<1\right\}
$$

shows. Namely any uniform limit of elements of $\mathcal{O}(\bar{D})$ will be holomorphic at $(0,0) \in \bar{D}$, but

$$
\begin{aligned}
f(z, w) & =w^{2} / z, & & z \neq 0, \\
& =0, & & z=0,
\end{aligned}
$$

defines an element of $\mathcal{O}(D) \cap C^{0}(\bar{D})$ which is not holomorphic at $(0,0)$.

However, it is unknown to us whether there are any examples $D$ with a smooth boundary such that (6.6) is not a dense map. We have a partial result in this direction, and for simplicity we restrict our attention to domains in $C^{n}$, since the general situation is somewhat more complicated, and not instructive with respect to the still unsolved problem in $\boldsymbol{C}^{n}$.

THEOREM 6.4. Let $D \subset \subset C^{n}$ be such that there is a domain $D^{\prime} \supset \supset D$ and a $C^{\infty}$ plurisubharmonic function $\varphi$ defined in $D^{\prime}$ with

(i) $D=\left\{x \in D^{\prime}: \varphi(x)<0\right\}$.

(ii) $d \varphi \neq 0$ on $\partial D$.

Then the natural restriction map $\mathcal{O}(\bar{D}) \rightarrow \mathcal{O}(D) \cap C^{\infty}(\bar{D})$ is dense. 
Proof. First we observe that any $f \in \mathcal{O}(D) \cap C^{\infty}(\bar{D})$ is the restriction to $\bar{D}$ of an $\tilde{f} \in C^{\infty}\left(D^{\prime}\right)$ such that $\bar{\partial} f$ vanishes to any prescribed order on $\partial D$ (see [8, p. 32]). Note that $\bar{\partial} \bar{f}$ is necessarily identically zero in $D$ since $\tilde{f}|D=f| D \in \mathcal{O}(D)$. Secondly the domains $D(\varepsilon)=\left\{x \in D^{\prime}: \varphi(x)<\varepsilon\right\}$ are, for $\varepsilon$ small and positive, pseudoconvex domains with $D \subset \subset D(\varepsilon)$, and $\bigcap_{\varepsilon>0} D(\varepsilon)=\bar{D}$. Thus we can solve the equation $\bar{\partial} u_{\varepsilon}=\bar{\partial} \bar{f}$ with an $L^{2}$ estimate

$$
\left\|u_{\varepsilon}\right\|_{D(\varepsilon)} \leqq K\|\bar{\partial} \tilde{f}\|_{D(\varepsilon)} \leqq K\left\|\bar{\partial}{ }^{\prime}\right\|_{D(\varepsilon)-D}
$$

since $\bar{\partial} \tilde{f}=0$ on $D$. Then using (5.1), the fact that $\bar{\partial} \tilde{f}$ vanishes to any prescribed order on $\partial D$, and Sobolev's lemma (as in (6.3)), we can make the uniform norm of $u_{\varepsilon}$ arbitrarily small on $\partial D$. Thus $\tilde{f}-u_{\varepsilon}$ will be the desired approximation. Q.E.D.

REMARK. Suppose $D$ is as in the above theorem, then let $\bar{\partial}_{b}$ denote the restriction of $\bar{\partial}$ to $\partial D$ (see [9]). If $f \in \mathcal{O}(D) \cap C^{0}(\bar{D})$ then $f \mid \partial D$ will be a continuous weak (in $L^{2}(\partial D)$ ) solution to the equation

$$
\bar{\partial}_{b} u=0 \text { on } \partial D \text {. }
$$

It is known that weak solutions of (6.7) are not necessarily $C^{\infty}$, but it is not known whether a continuous weak solution of (6.7), such as $f \mid \partial D$, can be uniformly approximated on $\partial D$ by some $g \in C^{\infty}(\partial D)$ which satisfies (6.7). If this were possible, then, it follows from Bochner's generalization of Hartog's theorem (see [8, p. 31]), that $g$ is the trace of a holomorphic function in $D$. Thus, by virtue of Theorem 6.3, this would show that (6.6) is dense for this class of domains.

We do not know whether an approximation theorem holds for general compact smooth real hypersurfaces, i.e., whether we can get rid of the restriction of having a plurisubharmonic function as in Theorem 6.4. Nevertheless, we can prove approximation locally:

THEOREM 6.5. Let $M$ be a real $C^{\infty}$ hypersurface defined in an open set $U \subset C^{n}$, and let $p \in M$. Then there is a fundamental system of compact neighborhoods $\mathscr{K}=\{K\}$ in $M$ of $p$ such that if $K \in \mathscr{K}$, then $A(K)=A_{M}(K)$.

Proof. With the notation of $\S 3$, we set $\mathscr{K}=\{K(\varepsilon)\}=\left\{\bar{W}_{T_{p}}(\varepsilon) \cap M\right\}$, for $\varepsilon>0$ small enough. If now $M=\{\rho=0\}$ near $p$, where $\rho$ is a $C^{\infty}$ real valued function defined on a neighborhood of $M$, with $\operatorname{grad} \rho \neq 0$ on $M$, then as in Lemma 3.3, and its proof there are $\varepsilon_{0}, \eta_{0}>0$ such that if $0<\varepsilon<\varepsilon_{0}$ and if $f$ is a CR function on $K=K(\varepsilon)$, we can find an extension $u \in C^{\infty}\left(U\left(\varepsilon, \eta_{0}\right)\right)$ of $f$ such that

$$
\bar{\partial} u=g \bar{\partial}_{\rho} \quad \text { on } U\left(\varepsilon, \eta_{0}\right),
$$

where $g \in C^{\infty}\left(U\left(\varepsilon, \eta_{0}\right)\right)$ vanishes to any prescribed order on $K$.

The trick is now to pick a function $\zeta_{t}(x) \in C^{\infty}(\boldsymbol{R})$ such that

(i) $\zeta_{t}(x)=1$ for $|x| \leqq t$;

(ii) $\zeta_{t}(x)=0$ for $|x|>2 t$;

(iii) $0 \leqq \zeta_{t}(x) \leqq 1$ for every $x$;

(iv) $\left|D^{m} \zeta_{t}(x)\right| \leqq C t^{-m}$ for some constant $C$. 
We then define the $(0,1)$ forms on $U\left(\varepsilon, \eta_{0}\right)$

$$
\alpha_{t}=\zeta_{t}(\rho) \bar{\partial} u=\zeta_{t}(\rho) g \bar{\partial} \rho .
$$

It can be easily seen that $\bar{\partial} \alpha_{t}=0$, and that the $L^{2}$ norm of $\alpha_{t}$ and of its derivatives up to order $m$ on $U\left(\varepsilon, \eta_{0}\right)$, can be made arbitrarily small by taking the order of vanishing of $g$ in (6.8) to be sufficiently high (this order depends only on $m$ ), and then making $t$ small enough. But then, as before, we can solve the $\bar{\partial}$-Neumann problem $\bar{\partial} v_{t}=\alpha_{t}$, on $U\left(\varepsilon, \eta_{0}\right)$ (which is a pseudoconvex domain), and by the estimates in $\S 5$, and Sobolev's lemma we see that $\left|v_{t}\right|_{K, 0}$ can be made arbitrarily small by taking $t$ to be small. But $\alpha_{t}=\bar{\partial} u$ on a neighborhood of $K(\varepsilon)$, therefore $u-v_{t}$ is holomorphic on this neighborhood, and provides the required approximation of $f$ on $K(\varepsilon)$. Q.E.D.

We are unable to prove a similar local result for lower dimensional CR manifolds, for we cannot find closed forms $\alpha_{t}$ with small support and which coincide with $\bar{\partial} u$ on a neighborhood of the manifold. But of course, this is not necessary when we have arbitrarily small pseudoconvex neighborhoods of $M$. Thus, we can prove

THEOREM 6.6. Let $M$ be a CR submanifold of an open set $U \subset C^{n}$, and suppose that the Levi form $\left({ }^{4}\right)$ of $M$ vanishes identically on a neighborhood of $p \in M$. Then there is a fundamental system of compact neighborhoods $\mathscr{K}=\{K\}$ in $M$ of $p$ such that if $K \in \mathscr{K}, A(K)=A_{M}(K)$.

Proof. With the notation of $\S 3$, if on $M(\varepsilon)=M \cap W_{T_{p}}(\varepsilon)$ the Levi form is identically 0 (which is true for $\varepsilon>0$ small enough), and if $M_{\tau}(\varepsilon)$ denotes a normal translate of $M(\varepsilon)$, then, setting

$$
W(\varepsilon, \eta)=\bigcup_{|\tau|<\eta} M_{\tau}(\varepsilon)
$$

it is proved in [16, Theorem 2.4] that $W(\varepsilon, \eta)$ is pseudoconvex for $\eta>0$ small enough. The remainder of the proof consists in extending the given function suitably to $W(\varepsilon, \eta)$, solving a $\bar{\partial}$-Neumann problem on $W(\varepsilon, \eta)$ and using the estimates in $\S 5$ and Sobolev's lemma. We omit the details as they follow the same lines as in the proof of Theorem 6.1. Q.E.D.

7. Applications to problems in global or polynomial approximation. In this section we shall combine some of the results in $\$ 6$ with standard results of several complex variables to yield some general theorems on approximation by globally defined functions, for example, polynomials in $C^{n}$. As special cases we obtain previously known results of various authors.

Let $K$ be a compact subset of a Stein manifold $X$, then we define the $\mathcal{O}(X)$-hull of $K$ to be

$$
\hat{K}_{X}=\left\{x \in X:|f(x)| \leqq \sup _{K}|f|, f \in \mathcal{O}(X)\right\}
$$

(4) See [16]; this is equivalent to assuming $M$ is a fibred family of complex submanifolds of $\boldsymbol{C}^{n}$. 
and we say that $K$ is $\mathcal{O}(X)$-convex if $K=\hat{K}_{X}$. In case $X=C^{n}, \mathcal{O}(X)$-convexity coincides with polynomial convexity $\left({ }^{5}\right)$. Note that there is a homeomorphism of $S(A(K, X))$ onto $\hat{R}_{X}$ [4, p. 213], and thus if $A(K, X)=C(K)$, it follows trivially that $K$ is $\mathcal{O}(X)$-convex. The converse is not true in general, but under additional assumptions on $K$, one can sometimes prove the converse, as we see in Theorem 7.3. First we shall see that a totally real submanifold of a Stein manifold admits local approximation by globally defined functions.

THEOREM 7.1. Let $M$ be a totally real $C^{\infty}$ submanifold of an open set $W$ in a Stein manifold $X$. Then for any point $p \in M$, there is a neighborhood $V$ of $p$, with $V \subset \subset W$, such that, if we set $K=\bar{V} \cap M$, then $A(K, X)=C(K)$, and consequently $K$ is $\mathcal{O}(X)$-convex.

Proof. Suppose $N \subset \subset M$ is a relatively compact subdomain of $M$ with a $C^{\infty}$ boundary such that $p \in N$. Then $\bar{N}$ is a finite totally real submanifold of $X$, and consequently, by Theorem 6.1 , there is an open set $U \subset X$ such that $U \supset \bar{N}$ and $\mathcal{O}(U)$ is dense in $C(\bar{N})$. Each point $q \in X$ has the property that there is a fundamental neighborhood system of $\mathcal{O}(X)$-convex subdomains of $X$, so we may suppose that $V$ and $B$ are $\mathcal{O}(X)$-convex domains in $X$ and $p \in V \subset \subset B \subset \subset U$. We shall show now that if we set $K=\bar{V} \cap M$, then $\mathcal{O}(X)$ is dense in $C(K)$. Namely, suppose $f \in C(K)$, then $f$ extends to an $\tilde{f} \in C(\bar{N})$ such that $\tilde{f} \mid K=f$. We can approximate $\tilde{f}$ on $\bar{N}$ by some $g \in \mathcal{O}(U)$, since $\mathcal{O}(U)$ is dense in $C(\bar{N})$. But $g \mid B \in \mathcal{O}(B)$ and approximates $f$ on $K$. Using the fact that $\mathcal{O}(X)$ is dense in $\mathcal{O}(B)$, since $B$ is $\mathcal{O}(X)$-convex, we obtain an $h \in \mathcal{O}(X)$ which approximates $f$ on $K$ as closely as desired. Q.E.D.

COROLLARY 7.2. If $M$ is a totally real $C^{\infty}$ submanifold of an open set $W$ in $C^{n}$, then for any point $p \in M$, and for any sufficiently small ball $B$ about $p$,

$$
A\left(\bar{B} \cap M, C^{n}\right)=C(\bar{B} \cap M),
$$

and $\bar{B} \cap M$ is polynomially convex.

Suppose $\Delta=\{\zeta \in C:|\zeta| \leqq 1\}$ is the closed unit disc in $C$, and suppose that $f \in C^{\infty}(\Delta)$, then the graph of $f$ in $C^{2}$ defines a diffeomorphic image $K$ of $\Delta$. Suppose that $f_{\bar{z}}(0) \neq 0$, then it follows immediately that $K$ is a submanifold of $C^{2}$ which is totally real near $p=(0, f(0))$, and we can apply Corollary 7.2 to conclude that near $p$ continuous functions on $M$ can be approximated by polynomials, and $K$ is locally polynomially convex at $p$. This result was obtained in this case by Wermer in [20]. A second result of Wermer in this direction states that if $f_{\bar{z}}(z) \neq 0$ at any point $z \in \Delta$, and if $K$ is polynomially convex, then $A\left(K, C^{n}\right)=C(K)$ (see [21]). This can also be easily generalized as follows:

THEOREM 7.3. Let $M$ be a compact or finite totally real $C^{\infty}$ submanifold of a Stein manifold $X$. Suppose $M$ is $\mathcal{O}(X)$-convex, then $A(M, X)=C(M)$.

${ }^{5}$ ) Sometimes $\mathcal{O}(X)$-convexity has been called "holomorphic convexity of $K$ in $X$ ", which we feel is ill-chosen terminology. 
Proof. Any $\mathcal{O}(X)$-convex compact subset of $X$ has a fundamental neighborhood system of relatively compact $\mathcal{O}(X)$-convex subdomains of $X$. Let $U$ be given by Theorem 6.1, such that $\mathcal{O}(U)$ is dense in $C(M)$, then find a subdomain $V$ of $X$ such that $M \subset \subset V \subset \subset U$, and such that $V$ is $\mathcal{O}(X)$-convex. Then use the fact that $\mathcal{O}(X)$ is dense in $\mathcal{O}(V)$, and the theorem is proven. Q.E.D.

When $X=C^{n}$, then $\mathcal{O}(X)$-convexity is the same as polynomial convexity, so we have the following corollary which contains Wermer's result in [21].

COROLlaRY 7.4. Let $M$ be a compact or finite totally real $C^{\infty}$ submanifold of $C^{n}$. If $M$ is polynomially convex, then $A\left(M, C^{n}\right)=C(M)$.

We remark that Corollary 7.4 contains the result of Helson and Quigley [5] for closed $C^{\infty}$ curves, since the converse of Theorem 7.3 is trivially true. Conversely, one can use [5] to prove Theorem 6.1 in the case of a closed $C^{2}$ curve embedded in $C^{n}$, by using the proper embedding theorem for Stein manifolds, the solution to the Levi problem $\left({ }^{6}\right)$, and Corollary 4.2 .

One could ask at this point whether one can remove the hypothesis of $\mathcal{O}(X)$ convexity in Theorem 7.3. Wermer has provided us with the following example which shows that this is not possible, in general. What we shall do is produce a function $f \in C^{\infty}(\Delta)$, so that $f_{\tilde{z}} \neq 0$ at all points of $\Delta$, and such that the graph of $f$, which we have called $K$, is not polynomially convex. To do this it is easy to see that it suffices to find a function $f \in C^{\infty}(\Delta)$ such that

(i) $f_{\bar{z}}(z) \neq 0$ on $\Delta$,

(ii) $f \mid \partial \Delta=0$.

Let $f(z)=(1+i) \bar{z}-i z \bar{z}^{2}-z^{2} \bar{z}^{3}$, and (i) and (ii) are satisfied.

However it is known that a compact differentiable arc $\Gamma$ in $\boldsymbol{C}^{n}$ (which is then a finite totally real submanifold) is polynomially convex (see [19]), and consequently by Corollary $7.4 A\left(\Gamma, C^{n}\right)=C(\Gamma)$, which is a contrast to the higher dimensional situation.

\section{BIBLIOGRAPHY}

1. H. J. Bremermann, Die Charakterisierung Rungescher Gebiete durch plurisubharmonische Funktionen, Math. Ann. 136 (1958), 173-186.

2. M. Freeman, "Some conditions for uniform approximation on a manifold" in Function algebras, Scott, Foresman \& Co., Chicago, Ill., 1965, pp. 42-65.

3. H. Grauert, On Levi's problem and the imbedding of real-analytic manifolds, Ann. of Math. 68 (1958), 460-472.

4. R. C. Gunning and H. Rossi, Analytic functions of several complex variables, Prentice-Hall, Englewood Cliffs, N. J., 1962.

5. H. Helson and F. Quigley, Maximal algebras of continuous functions, Proc. Amer. Math. Soc. 8 (1957), 111-114.

6. L. Hörmander, Linear partial differential operators, Springer-Verlag, Berlin, 1963.

${ }^{(6)}$ This was pointed out to us by $\mathrm{H}$. Alexander, and is contained in the thesis of $\mathrm{H}$. Rossi (unpublished). 
7. L. Hörmander, $L^{2}$ estimates and existence theorems for the $\bar{\partial}$ operator, Acta Math. 113 (1965), 89-152.

8. - An introduction to complex analysis in several variables, Van Nostrand, Princeton, N. J., 1966.

9. J. J. Kohn, "Boundaries of complex manifolds" in Proceedings of the conferences on complex analysis, Minneapolis, 1964, Springer-Verlag, Berlin, 1965, pp. 81-94.

10. J. J. Kohn and L. Nirenberg, Non-coercive boundary value problems, Comm. Pure Appl. Math. 18 (1965), 443-492.

11. J. Milnor, Lectures in differential topology, Princeton Univ., Princeton, N. J., 1958 (mimeographed).

12. R. Nirenberg and R. O. Wells, Jr., Holomorphic approximation on real submanifolds of a complex manifold, Bull. Amer. Math. Soc. 73 (1967), 378-381.

13. G. Stolzenberg, Uniform approximation on smooth curves, Acta Math. 115 (1966), $185-198$.

14. R. O. Wells, Jr., On the local holomorphic hull of a real submanifold in several complex variables, Comm. Pure Appl. Math. 19 (1966), 145-165.

15. - Holomorphic approximation on real-analytic submanifolds of a complex manifold, Proc. Amer. Math. Soc. 17 (1966), 1272-1275.

16. - Holomorphic hulls and holomorphic convexity of differentiable submanifolds, Trans. Amer. Math. Soc. 132 (1968), 245-262.

17. - Compact differentiable submanifolds of a complex manifold with a nondegenerate holomorphic tangent bundle, Math. Ann. 179 (1969), 123-129.

18. - Real-analytic subvarieties and holomorphic approximation, Math. Ann. 179 (1969), 130-141.

19. J. Wermer, Uniform approximation and maximal ideal spaces, Bull. Amer. Math. Soc. 68 (1962), 298-305.

20. —-, Approximation on a disk, Math. Ann. 155 (1964), 331-333.

21. - Polynomially convex disks, Math. Ann. 158 (1965), 6-10.

22. L. Hörmander and J. Wermer, Uniform approximation on compact subsets in $C^{n}$, (to appear).

BRANDEIS UNIVERSITY,

Waltham, MassachusetTs

RICE UNIVERSITY,

Houston, TeXas 